\title{
POTENTIALITÉS DE L'ALISIER TORMINAL SUR SOLS À HYDROMORPHIE TEMPORAIRE : COMPARAISON AVEC LES CHÊNES PÉDONCULÉ ET SESSILE
}

\author{
G. LÉVY - N. LE GOFF - Sabine GIRARD - Y. LEFÈVRE
}

Cet article fait suite au stage de DEA de Biologie végétale et forestière (Université de Nancy l) effectué par Sabine Girard en 1990-1991 à la Station de Sylviculture et de Production et à la Station de Recherches sur le Sol, la Microbiologie et la Nutrition des Arbres forestiers du Centre de Recherches de I'INRA à Nancy.

Les sols à hydromorphie temporaire sont très répandus en forêt dans de nombreuses régions françaises. Les peuplements y sont généralement soumis à deux contraintes hydriques successives au cours d'une même saison de végétation. D'une part, la présence d'une nappe d'eau temporaire remontant jusqu'à proximité de la surface du sol à certaines périodes, situées le plus souvent en hiver et au début du printemps, crée des conditions hypoxiques qui rendent difficile la respiration des racines et perturbent donc leur fonctionnement. D'autre part, la sécheresse estivale peut créer des déficits hydriques particulièrement marqués sur ces sols, car la nappe printanière réduit le volume de sol prospecté par les racines, donc la réserve d'eau qui sera à leur disposition en été (Belgrand, 1983). Chacun de ces deux stress est plus ou moins intense selon les conditions climatiques de l'année considérée.

Les pélosols-pseudogleys, qui sont très répandus, notamment dans l'Est de la France, sont des sols hydromorphes sur lesquels ces deux contraintes sont particulièrement marquées. Le plancher de la nappe apparaît en effet à faible profondeur (au maximum à $30 \mathrm{~cm}$ de la surface du sol), et il possède une texture très argileuse; les nappes sont donc souvent très superficielles, ennoyant alors la totalité du système racinaire des arbres.

Les peuplements forestiers situés sur pélosol-pseudogley sont généralement à base de Chêne pédonculé, et se caractérisent par une faible productivité (Belgrand et Lévy, 1985). Le Chêne sessile peut également s'y trouver, bien que beaucoup plus rarement. Les recherches effectuées dans le passé par l'INRA ont permis de proposer au gestionnaire un certain nombre d'essences résineuses relativement bien adaptées à ces sols (Claude, 1986), mais aucune étude de terrain n'avait porté jusqu'à présent sur des espèces feuillues autres que le Chêne pédonculé. Or, I'Alisier torminal, dont on connaît les excellentes propriétés technologiques du bois (Lanier et al., 1990), est présent sur pélosol-pseudogley (Jacamon, 1984), mais nous ne connaissons pas ses potentialités sur ces sols, ni sa sensibilité aux deux contraintes hydriques successives qui les caractérisent. 


\section{G. LÉVY - N. LE GOFF - Sabine GIRARD - Y. LEFÈVRE}

Aussi, avons-nous étudié les variations de croissance de l'Alisier sur une gamme de sols formés sur argile ou marnes du Secondaire, recouvertes ou non d'une couche plus ou moins épaisse de limons, et englobant les pélosols-pseudogleys. Les deux contraintes hydriques sont plus ou moins intenses selon le type et les propriétés de ces sols. Le comportement de l'Alisier a été comparé à celui du Chêne pédonculé, qui a servi de référence. La méthodologie utilisée a permis par ailleurs d'intégrer le Chêne sessile à l'étude.

Le travail réalisé diffère des études classiques de relations station-production (Garbaye et al., 1970) en raison de plusieurs problèmes spécifiques au matériel d'étude disponible. Tout d'abord, l'Alisier torminal est une espèce disséminèe dans les peuplements de Chêne (individus isolés ou par groupes de deux ou trois arbres) et ne constitue donc pas de populations que l'on puisse caractériser par des grandeurs moyennes (telle la hauteur dominante). Ensuite, les alisiers se rencontrent le plus fréquemment en taillis-sous-futaie, type de peuplement où les traitements sylvicoles influencent la croissance en hauteur des arbres (Le Goff, 1982 ; Pardé et Bouchon, 1988 , p. 221), alors que les indices dendrométriques rendant compte de la fertilité des stations se basent le plus souvent sur cette croissance en hauteur (Décourt, 1973; Franc et Houllier, 1989 ; Duplat, 1989).

Ne pouvant disposer, de plus, que de données instantanées pour caractériser la croissance des arbres (couples hauteur/âge), la méthodologie d'étude a été adaptée de celle utilisée pour étudier les potentialités d'espèces disséminées en taillis-sous-futaie à partir de mesures instantanées, sur la base d'une typologie des stations (Le Goff et Madesclaire, 1989). Pour cela, une typologie des sols, liée aux facteurs limitants de la croissance des arbres sur ces sols, a été réalisée (cf. ci-après).

Ce sont essentiellement les variables hydriques qui constituent les facteurs limitants de la croissance des arbres dans la gamme de stations étudiée, en particulier les conditions d'approvisionnement en eau. Or cet approvisionnement dépend notamment de la profondeur d'enracinement des arbres, donnée très difficile à déterminer.

En raison de toutes ces difficultés, il ne faut pas attendre de cette étude des résultats très précis ni très approfondis. Cependant, les connaissances actuelles dans ce domaine étant excessivement réduites, elle devrait permettre de fournir des données nouvelles sur l'écologie comparée sur sols hydromorphes des trois espèces prises en compte, et en particulier sur celle de l'Alisier torminal, ainsi que sur la mise en valeur possible des sols de la gamme étudiée.

\section{MATÉRIEL ET MÉTHODES}

\section{Région d'étude}

La prospection a été réalisée sur le Plateau lorrain, dans des forêts dont le substrat géologique était susceptible de convenir pour l'étude. Nous avons finalement retenu trois massifs forestiers où l'Alisier est relativement abondant et qui présentent une variété importante de sols faisant partie de la gamme recherchee: Parroy (Meurthe-et-Moselle), Charmes-Damas-aux-Bois (Vosges), et Saint-Vallier - Bouxières-aux-Bois (Vosges). Le substrat de l'époque Secondaire est constitué par les marnes du Keuper inférieur, que l'on retrouve dans toutes les placettes choisies.

\section{Choix des placettes d'étude}

Les placettes devaient couvrir une surface suffisamment faible pour être homogènes sur le plan écologique (sol, topographie) et comprendre au moins un alisier et si possible deux chênes. Les peuplements retenus devaient tous être des taillis-sous-futaie, au traitement sylvicole le plus 
semblable possible; un contrôle des conditions de densité actuelle et passée a pour cela été effectué. Les arbres choisis devaient appartenir à l'étage dominant et posséder un houppier bien développé et équilibré.

73 placettes ont été finalement retenues pour l'étude, dont 17 à Parroy, 36 à Charmes - Damas et 20 à Saint-Vallier - Bouxières.

\section{Recueil des données}

La hauteur totale, la hauteur de la base du houppier et l'âge ont été mesurés sur chacun des arbres retenus. Les études écologiques permettant de caractériser chaque placette ont porté sur la situation topographique, les sols (description et analyse des propriétés physiques et chimiques) et la fluctuation des nappes.

\section{Indice de fertilité}

Dans les études de relations station-production classiques en futaie pure et équienne, l'indice de fertilité, constitué par la hauteur dominante du peuplement à un âge de référence, peut aussi être considéré le plus souvent comme indice de productivité (Duplat, 1989). En taillis-sousfutaie, des indices de fertilité ou de productivité pour l'espèce principale constituant la futaie ont pu être élaborés à l'échelle du peuplement (Le Goff, 1984 ; Doussot et Grandjean, 1989). Mais les méthodes développées ne sont pas adaptées pour de petites placettes d'étude ni pour des espèces disséminées comme l'Alisier. Un indice de fertilité a cependant pu être élaboré pour les Chênes comme pour l'Alisier, basé sur la croissance en hauteur de chaque espèce, caractéristique de chaque type de sols défini, en tenant compte des effets éventuels des variations dans le traitement sylvicole des peuplements.

Dans ce travail-ci, la hauteur mesurée est apparue indépendante de l'âge, probablement parce que la croissance en hauteur était devenue négligeable en raison de l'âge atteint par les arbres retenus et du traitement en taillis-sous-futaie. La hauteur n'a donc pas été rapportée à un âge de référence déterminé.

II est apparu cependant pour deux des trois espèces étudiées, l'Alisier et le Chêne sessile, une relation linéaire entre la hauteur relative du houppier, variable liée aux conditions de concurrence passées, et la hauteur des arbres. Pour ces deux espèces, la hauteur des arbres a donc été corrigée (1) pour calculer l'indice de fertilité de l'espèce considérée sur chaque type de sol, afin de tenir compte des effets de conditions de concurrence variables dans l'ensemble des placettes. Pour le Chêne pédonculé, la hauteur mesurée a été retenue sans modification, en l'absence de relation entre la hauteur et les conditions de concurrence passées.

Nous avons émis l'hypothèse que la croissance en hauteur des arbres dépendait essentiellement, dans la gamme des types de station retenue, de leur réaction aux deux contraintes hydriques présentées ci-dessus. Or, l'intensité de ces contraintes dans chacune des placettes dépend de nombreuses variables stationnelles liées au sol et à la topographie, variables qui sont le plus souvent interdépendantes. C'est pourquoi nous avons retenu une typologie des sols permettant de représenter simplement et d'une manière synthétique les propriétés stationnelles importantes. La variation des indices de fertilité des trois espèces étudiées a donc ensuite été analysée en fonction des types de sol définis.

(1) La hauteur corrigèe $\left(\mathrm{H}_{\mathrm{c}}\right)$ d'un arbre est calculèe de la manière suivante:

$\mathrm{Ht}_{c}=\mathrm{Ht}+\mathrm{b}\left(\mathrm{HrH}_{\mathrm{m}}-\mathrm{HrH}\right)$

où : $\mathrm{Ht}=$ hauteur de l'arbre ;

$\mathrm{HrH}=$ hauteur relative du houppier de l'arbre;

$\mathrm{HrH}_{\mathrm{m}}=$ hauteur relative du houppier moyenne observée pour les arbres èchantillonnès de l'espèce considèrée

$b=$ pente de la droite ajustée pour rendre compte des variations de la hauteur avec la hauteur relative du houppier pour l'espéce considérèe. 


\section{G. LÉVY - N. LE GOFF - Sabine GIRARD - Y. LEFĖVRE}

\section{ÉTUDE DES RELATIONS ENTRE LES TYPES DE SOL ET L'INDICE DE FERTILITÉ DE L'ALISIER ET DES CHÊNES}

\section{Typologie des sols en accord avec les contraintes subies par les arbres}

Les résultats des analyses chimiques effectuées ont permis de vérifier que les sols concernés par cette étude étaient toujours suffisamment pourvus en élements nutritifs assimilables. Aussi, nous sommes-nous intéressés surtout à leurs propriétés physiques.

Nous avons essentiellement pris en compte dans cette typologie les variables individuelles dont dépend l'intensité de l'ennoyage du système racinaire des arbres: épaisseur éventuelle de la couche de limons recouvrant les argiles, caractéristiques de la zone de contact entre limons et argile (changement de texture brutal ou progressif), structure et sa stabilité, traces d'hydromorphie (confirmées par des mesures de fluctuation des nappes), pente. Ce sont d'ailleurs ces variables qui, parmi l'ensemble de celles qui ont ètè mesurées, sont généralement le plus liées aux indices de fertilité des trois espèces, bien que les coefficients de corrélation ne soient jamais très élevés.

Cinq types de sol ont été ainsi définis. On distingue, par ordre d'engorgement croissant du système racinaire des arbres : pélosols brunifiés, pseudogleys, pélosols-pseudogleys de type II, pélosols-pseudogleys de type I, pélosols brunifiés marmorisés (le terme "marmorisés " utilisé à la place de "à pseudogley" ne signifie pas que l'engorgement n'est pas drastique, mais simplement que les traces d'hydromorphie sont peu marquées sur ce type de sol, et cela en raison de sa teneur en matière organique et de sa texture). La figure $\uparrow$ (ci-dessous) représente les caractères distinctifs de ces sols.

Figure 1

TYPOLOGIE OES SOLS ÉTUDIÉS FORMÉS SUR ARGILES (OU MARNES) ET RECOUVERTS OU NON D'UNE COUCHE D'ÉPAISSEUR VARIABLE DE LIMONS : les types définis se caractérisent par une Intensité variable de l'ennoyage du système racinaire des arbres (engorgement croissant).

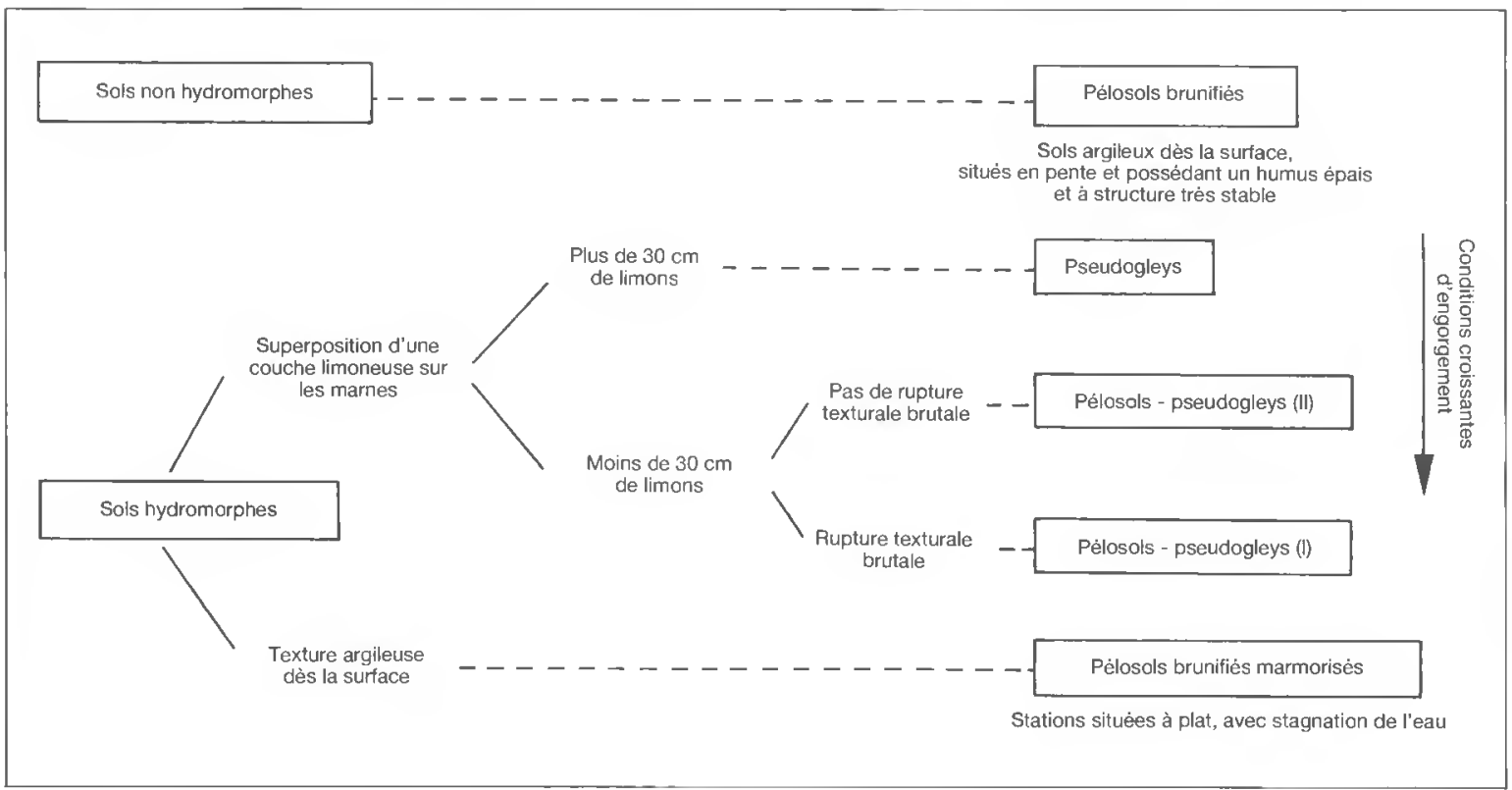


La contrainte "déficit hydrique " est également bien représentée dans cette typologie, bien qu'il soit plus difficile de la définir de façon précise dans chaque placette après une étude stationnelle. Elle est en effet bien liée, dans cette gamme de sols, à la contrainte " engorgement " sur laquelle est fondée la typologie. C'est ainsi que le niveau d'approvisionnement en eau des arbres dépend de la profondeur atteinte par leur système racinaire, profondeur bien en relation avec les conditions d'engorgement; par ailleurs, une texture très argileuse, d'une part favorise la remontée des nappes, et d'autre part constitue un milieu défavorable à la prospection des racines, donc à leur approvisionnement en eau. C'est ainsi que :

- Les pélosols brunifiés, bien qu'assez superficiels, ne sont pas des sols à nappe temporaire. Leur horizon $A_{1}$ est épais et possède une structure nette et stable. Les conditions d'approvisionnement en eau des arbres sur ces sols sont nettement plus favorables que sur pélosols brunifiés marmorisés, dont la texture est identique, mais qui sont souvent très engorgés, et que sur pélosols-pseudogleys, où l'argile apparaît à faible profondeur et où la nappe remonte souvent jusqu'en surface.

- Les pélosols brunifiés marmorisés et les deux types de pélosols-pseudogleys ne peuvent stocker que de très faibles réserves en eau utilisable.

- Sur pseudogley, où la couche de limons possède par définition une épaisseur supérieure à $30 \mathrm{~cm}$, la nappe est moins drastique et les horizons à texture favorable à l'enracinement sont plus épais que sur les trois sols précédents. Les conditions d'approvisionnement en eau des arbres sont ainsi bien plus propices, et cela d'autant plus que la couverture limoneuse est plus épaisse. Par ailleurs, la réserve maximum en eau utile des pseudogleys les plus superficiels est probablement inférieure à celle des pélosols brunifiés, alors que celle des pseudogleys les plus profonds lui est sans doute supérieure.

\section{Indices de fertilité et types de sol}

\section{- Cas de l'Alisier}

L'indice de fertilité de l'Alisier, pour un type de sol donné, est donc la hauteur moyenne corrigée des arbres échantillonnés appartenant au type considéré, calculée pour les conditions moyennes d'espace vital observées pour les alisiers (ce qui correspond à une hauteur relative moyenne du houppier égale à 0,531 ).

La figure 2 (p. 248) présente les variations de la hauteur corrigée de l'Alisier pour les différents types de sols définis, classés en fonction du degré d'engorgement. Malgré la relative faiblesse d'échantillonnage de certains types de sol (pélosol brunifiẻ et pseudogley), il apparaît que la croissance en hauteur de l'Alisier est meilleure sur pélosol brunifié que sur les autres types de sols [différences entre les moyennes de distribution significatives au seuil de $5 \%$ (cf. Parker, 1979) ]. Par contre, la croissance en hauteur sur les différents types de sols hydromorphes n'est pas significativement différente. L'indice de fertilité atteint ainsi $20,5 \mathrm{~m}$ sur les pélosols brunifiés contre $18 \mathrm{~m}$ environ pour l'ensemble des sols hydromorphes.

\section{- Cas des Chênes comparé à celui de l'Alisier}

Comme pour l'Alisier, les hauteurs des Chênes ont été corrigées pour tenir compte de l'effet des traitements sylvicoles passés sur la croissance en hauteur (effet significatif seulement pour le Chêne sessile). L'indice de fertilité pour les deux espèces de Chênes a donc été calculé de la même façon que pour l'Alisier, en corrigeant éventuellement les hauteurs (cas du Chêne sessile).

La figure 3 (p. 249) présente les variations comparées de l'indice de fertilité de l'Alisier, du Chêne pédonculé et du Chêne sessile en fonction du type de sol; cet indice représente la hauteur moyenne des arbres échantillons, pour les conditions moyennes d'espace vital des 


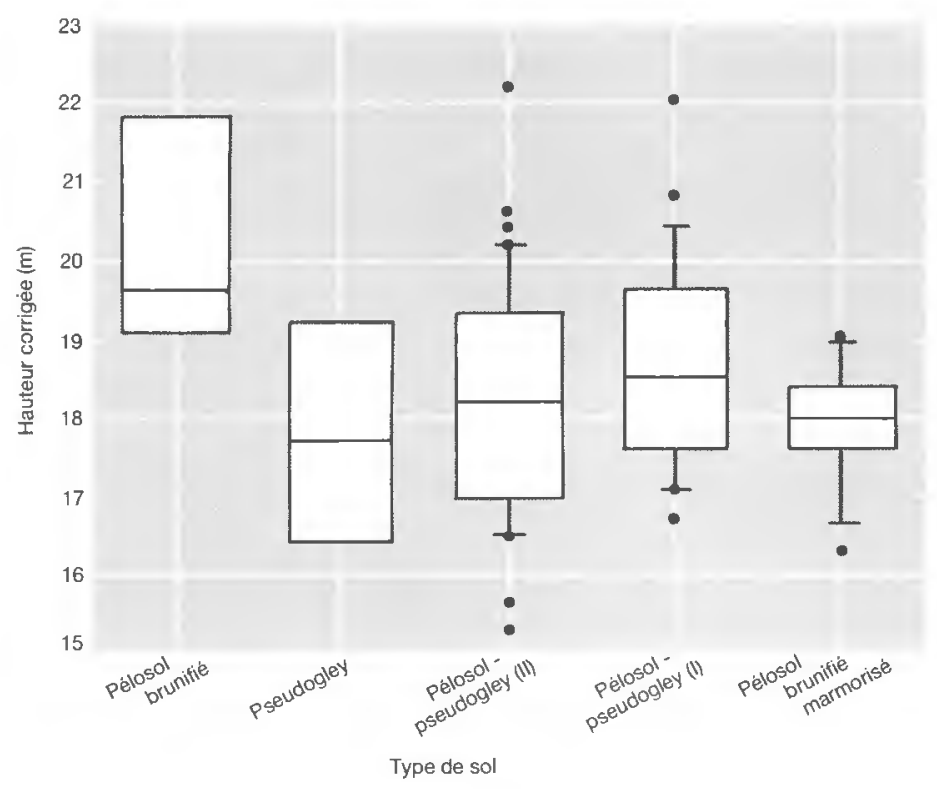

Figure 2

DISTRIBUTIONS RÉSUMÉES

DES HAUTEURS CORRIGÉES

DES ALISIERS POUR LES 5 TYPES

DE SOLS IDENTIFIÉS :

la représentation de chaque

distribution ( box plot ) fait

notamment apparaître sous

forme de rectangle la partie

centrale de la disfribution

comportant $50 \%$ des

observations ainsi que la valeur

médiane sous forme d'un fraif

qui partage ce rectangle

(Chambers et al., 1983).

arbres propres à chaque espèce (2). La croissance en hauteur du Chêne sessile apparait ainsi toujours supérieure à celle du Chêne pédonculé, sauf sur pélosols brunifiés marmorisés, et à celle de l'Alisier. Celle du Chêne pédonculé est quant à elle supérieure à celle de l'Alisier, sauf sur les pélosols brunifiés.

On remarque par ailleurs que c'est sur les sols les moins hydromorphes que la croissance des trois espèces est la meilleure: pélosol brunifié et encore davantage pseudogley pour les Chênes, seulement pélosol brunifié (seul sol non hydromorphe) pour l'Alisier.

\section{DISCUSSION ET CONCLUSION}

\section{Interprétation du comportement des trois espèces}

\section{- Cas des Chênes}

De précédentes études ont établi que le chêne pédonculé supporte relativement bien un engorgement temporaire superficiel du sol (Becker et Lévy, 1990), mais qu'il est très sensible à la sécheresse (Becker et Lévy, 1983). Un engorgement superficiel est au contraire le facteur limitant essentiel de la croissance du Chêne sessile (Keime, 1985), tandis que les déficits hydriques affectent beaucoup moins sa croissance que celle du Chêne pédonculé (Keime, 1985 ; Lévy et al., 1992).

Les résultats obtenus lors de cette étude peuvent être interprétés sur la base de ces caractéristiques écologiques. Ainsi, la croissance du Chêne sessile est la plus mauvaise sur les sols présentant l'engorgement le plus superficiel (pélosols-pseudogleys de type I et surtout pélosols brunifiés marmorisés). Pour le Chêne pédonculé, la croissance est médiocre sur les sols qui

(2) La hauteur relative moyenne du houppier observèe $(0,53$ pour les Alisiers, 0,48 pour les Chẻnes pédonculés et 0,47 pour les Chênes sessiles) tendrait à montrer que les conditions de concurrence moyennes subies par les arbres èchantillonnès diffèrent peu d'une espèce à l'autre. 
possèdent une réserve en eau utile très faible, en raison d'une part de leur texture très argileuse et d'autre part du fait que la nappe réduit la prospection du sol par les racines (pélosolspseudogleys et pélosols brunifiés marmorisés). Les arbres sont ainsi exposés sur ces sols à des déficits hydriques sévères au cours des périodes sèches.

Le comportement relatif des deux espèces de Chêne sur sol hydromorphe formé sur argile recouverte de limons plus ou moins épais (pélosol-pseudogley, pseudogley) est ainsi comparable à ce qu'on observe sur pseudogley acide (Becker et Lévy, 1986): la meilleure résistance à la sécheresse du Chêne sessile prend globalement le pas sur la plus grande tolérance du Chêne pédonculé à l'hydromorphie. Par contre, dans le cas d'un plancher imperméable pratiquement superficiel (pélosol brunifié marmorisé), les etfets de la contrainte " hydromorphie " deviennent nettement plus limitants sur la croissance du Chêne sessile qui se trouve alors rattrapé par le Chêne pédonculé (figure 3 , ci-contre).

Figure 3

VARIATIONS DE L'INDICE DE FERTILITÉ DE L'ALISIER, DU CHÊNE PÉDONCULÉ ET DU CHÊNE SESSILE SUR LES 5 TYPES DE SOLS IDENTIFIES.

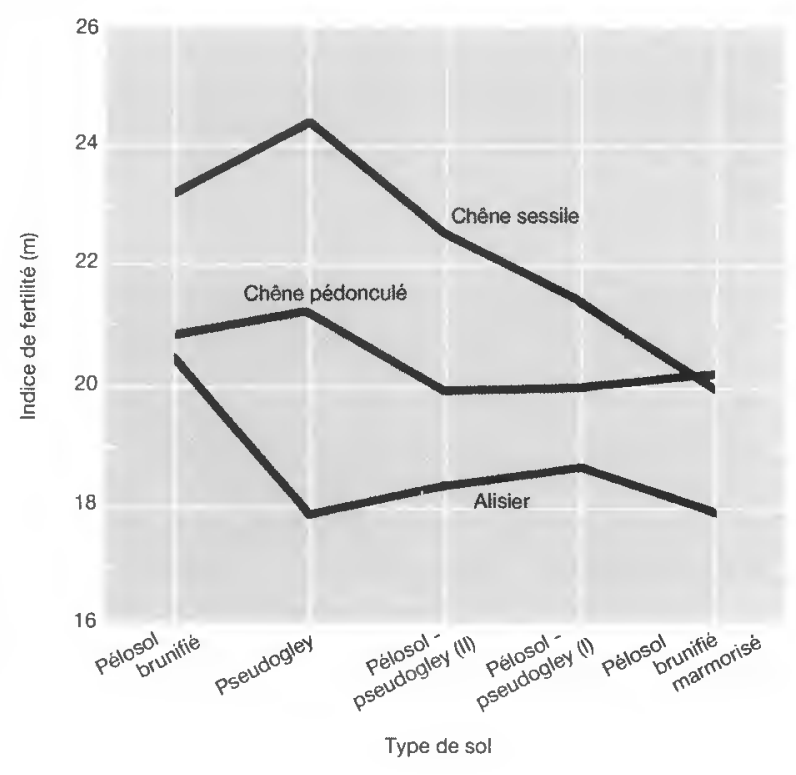

\section{- Cas de l'Alisier}

La bonne croissance relative de l'Alisier sur les pélosols non hydromorphes (englobant à la fois les pélosols sensu stricto et les pélosols brunifiés), qui a pu être mise en évidence lors de travaux antérieurs (Nicloux, 1988 ; Rameau, 1989), a été confirmée dans notre étude pour le cas des pélosols brunifiés, sols plus favorables que les pélosols sensu stricto car plus profonds. La croissance de l'Alisier étant meilleure sur ces types de sol que sur les sols hydromorphes, y compris sur les pseudogleys, on peut supposer que l'Alisier est surtout sensible à l'engorgement du sol. En effet, les pélosols brunifiés sont les seuls de la gamme étudiée qui ne présentent pas de nappe temporaire; par ailleurs, les conditions d'approvisionnement en eau des arbres n'y sont sans doute pas plus favorables que sur les pseudogleys considérés dans cette étude. Cette hypothèse de sensibilité de l'Alisier aux conditions d'engorgement du sol plutôt qu'à la sécheresse irait dans le sens des déductions sur le comportement de l'Alisier faites par Lachaud (1993) à partir de l'étude anatomique du boís qui révèle que l'Alisier présente des caractéristiques comparables à celles d'espèces résistant à la sécheresse.

Mise en valeur des sols formés sur argile recouverte ou non de limons. Perspectives de recherche

L'Alisier et les Chênes ne peuvent être comparés réellement dans le cadre de cette étude sur le plan de la production en volume, pour un certain nombre de raisons: faible représentation de l'Alisier dans les peuplements à base de Chêne, conditions de croissance passées de cette 
espèce en taillis-sous-futaie dominé par les chênes, influence sûrement insuffisamment contrôlée des traitements sylvicoles sur la croissance en hauteur. D'autre part, la comparaison des deux chênes entre eux peut laisser quelque peu à désirer; une étude orientée uniquement dans ce but permettrait de retenir un bien plus grand nombre de placettes d'étude comprenant les deux espèces.

Néanmoins, le comportement relatif des trois espèces sur les différents types de sols étudiés permet de faire certaines propositions quant au matériel à privilégier en fonction du type de sol. Pour cela, on a élaboré un indice de fertilité relatif (cf. Becker et Le Goff, 1988) en prenant l'indice de fertilité du Chêne sessile comme référence, cette espèce présentant la meilleure croissance sur presque tous les types de sol (cf. figure 3, p. 249). La figure 4 (ci-dessous) présente les variations de cet indice de fertilité relatif en fonction des types de sol pour l'Alisier et le Chêne pédonculé (l'indice prend la valeur 1 pour le Chêne sessile quel que soit le type de sol).

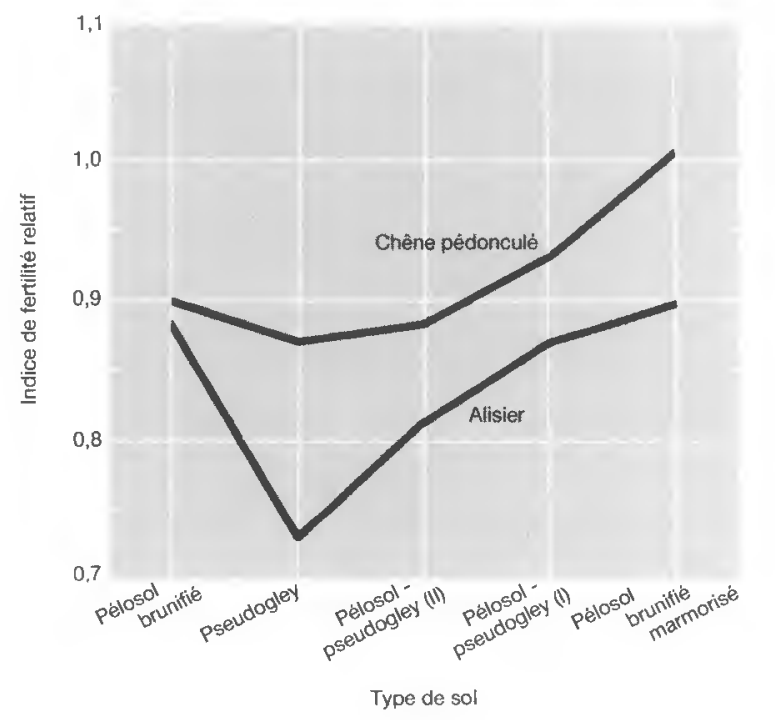

Figure 4

INDICES DE FERTILITÉ DE L'ALISIER ET DU CHÊNE PÉDONCULÉ COMPARÉS À CELUI DU CHÊNE SESSILE (PRIS COMME RÉFÉRENCE) SUR LES 5 TYPES DE SOLS IDENTIFIÉS.

En ce qui concerne l'Alisier, les tendances dégagées de l'analyse des variations de son indice de fertilité sont suffisamment claires pour établir que cette espèce est nettement moins adaptée que les chênes sur les sols hydromorphes. L'Alisier pourrait par contre être favorisé sur les pélosols non hydromorphes (brunifiés ou non), où sa croissance est satisfaisante et où il pourrait donc fournir des produits de bonne qualité actuellement très recherchés.

II conviendra d'autre part de favoriser le plus souvent le Chêne sessile sur les pélosolspseudogleys et les pseudogleys. Sur pélosol brunifié marmorisé, le Chêne pédonculé semble par contre mieux convenir: son indice de fertilité y est identique à celui du Chêne sessile, alors que la qualité de son bois est globalement supérieure à celle de cette dernière espèce (Lévy et al., 1992). Mais les conditions topographiques peuvent dans certains cas compenser des propriétés édaphiques défavorables: le Chêne pédonculé est relativement performant sur tous les sols de la gamme étudiée lorsque les conditions d'approvisionnement latéral en eau sont particulièrement favorables, en particulier en bas de pente et dans les vallons (Lévy et al., 1992).

Des études complémentaires, notamment de nature écophysiologique, réalisées aussi bien en plantation expérimentale qu'en conditions contrôlées, sont souhaitables en vue d'améliorer nos 
connaissances sur l'Alisier. Elles permettraient d'apprécier les réponses de cette espèce à l'engorgement du sol et à la sécheresse, ainsi qu'à la succession de ces deux contraintes au cours d'une même année, succession qui caractérise les sols à hydromorphie temporaire.
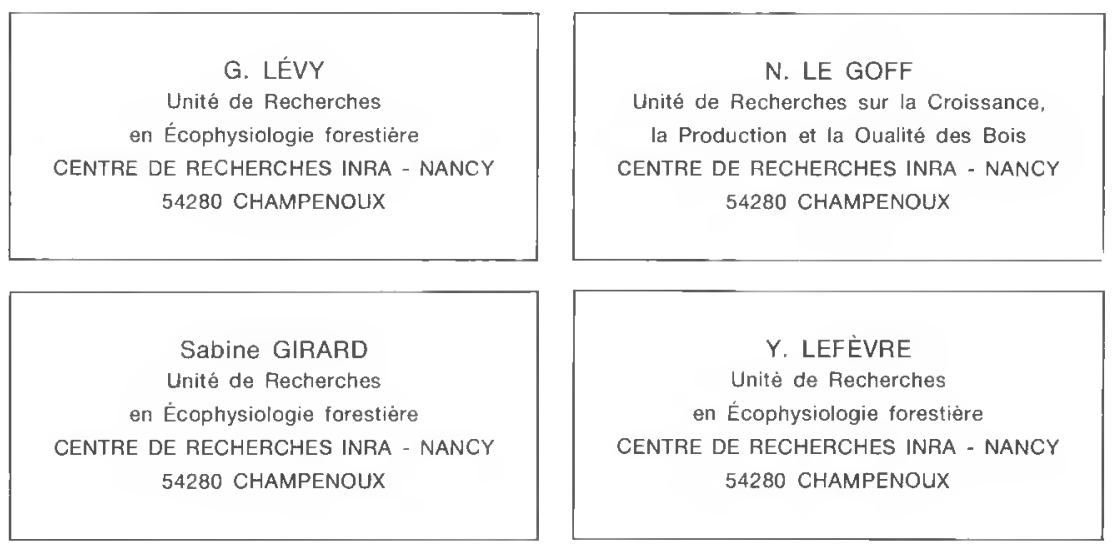

\section{Remerciements}

Les mesures dendrométriques effectuées sur le terrain pour cette étude ont été conduites par L. Garros, avec la collaboration technique de R. Canta, P. Garnier et G. Thiriet. Nous tenons aussi à remercier C. Marchal qui a contribué à la mise en ceuvre de cette étude en tant que stagiaire de DEA du Centre INRA de Nancy en 19891990. La prospection pour le choix des placettes d'observation doit beaucoup aux personnels de l'ONF des massifs forestiers concernés ainsi qu'à M. Rameau, professeur à l'ENGREF-Nancy. Cette étude a été réalisée dans le cadre du programme de recherches sur l'Alisier financé par la DERF et a également bénéficié d'aides de l'ONF, notamment pour le creusement des fosses pédologiques.

\section{BIBLIOGRAPHIE}

BECKER (M.), LE GOFF (N.). - Diagnostic stationnel et potentiel de production. - Revue forestière française, vol. XL, no spécial "Diagnostics en forêt ", 1988, pp. 29-43.

BECKER (M.), LÉVY (G.). - Comportement comparé de Chênes adultes (Quercus robur L. et Quercus petraea (Matt.) Liebl.) sur sol hydromorphe acide. Effet du drainage. - Acta Oecologica. Oecologia plantarum, vol. $21, n^{\circ} 2,1986$, pp. 121-143.

BECKER (M.), LÉVY (G.). - Le Dépérissement du Chêne. Les causes écologiques. Exemple de la forêt de Tronçais et premières conclusions. - Revue forestière française, vol. XXXV, $n^{\circ} 5,1983, p p .341-356$.

BECKER (M.), LÉVY (G.). - Le Point sur l'écologie comparée du Chêne sessile et du Chêne pédonculé. Revue forestiere française, vol. XLII, $\pi^{\circ} 2$ spécial "Chêne sessile, Chêne pédonculé et Chêne rouge d'Amérique en torẻt trançaise ", 1990, pp. 148-154.

BELGRAND (M.). - Comportement de jeunes plants feuillus (Chêne pédonculé, Chêne rouge, Chêne sessile, Hêtre) sur substrat ennoyé. Adaptations racinaires. Application à la mise en valeur forestière des pseudogleys. - Champenoux : INRA, 1983. - $188 \mathrm{p}$. + annexes (Thèse de Docteur-Ingénieur).

BELGRAND (M.), LEVY (G.). - Comportement de différentes essences forestières sur les sols à hydromorphie temporaire. - Science du Sol, $\mathrm{n}^{\circ} 4,1985, \mathrm{pp} .227-237$. 
CHAMBERS (J.M.), CLEVELAND (W.S.), KLEINER (B.), TUKEY (P.A.). - Graphical methods for data analysis. Bell Laboratories, Wadsworth International Group and Duxbury Press, 1983.

CLAUDE (S.). - Étude du comportement d'essences introduites sur sols à hydromorphie temporaire en Lorraine et en Haute-Saône. Relations hydromorphie-engorgement et sol-végétation. - Champenoux: INRA-Nancy, 1986. - 140 p. (Mémoire $3^{\mathrm{e}}$ annèe ENITEF).

DÉCOURT (N.). - Méthode utilisée pour la construction rapide des tables de production provisoires en France. - Annales des Sciences forestières, vol. 29, $\mathrm{n}^{\circ} 1,1972, \mathrm{pp} .35-48$.

DOUSSOT (R.), GRANDJEAN (G.). - Méthode d'appréciation de la productivité des stations forestières applicable aux taillis avec réserves et aux futaies régulières adultes. In : Station forestière, production et qualité des bois : éléments méthodologiques. - CEMAGREF, 1989. - $254 \mathrm{p}$.

DUPLAT (P.). - Indice de fertilité basé sur un modèle de croissance en hauteur. In: Station forestière, production et qualité des bois : éléments méthodologiques. - CEMAGREF, 1989. - $254 p$.

FRANC (A.), HOULLIER (F.). - Étude des relations entre milieu et production. Quelques critères de choix des méthodes. In: Station forestière, production et qualité des bois : éléments méthodologiques. - CEMAGREF, 1989. - $254 \mathrm{p}$.

GARBAYE (J.), LEROY (Ph.), LE TACON (F.), LÉVY (G.). - Réflexions sur une méthode d'études des relations entre facteurs écologiques et caractéristiques des peuplements. - Annales des sciences forestieres, vol. 27, $\mathrm{n}^{\circ} 3,1970$, pp. 303-321.

JACAMON (M.). - Guide de dendrologie. Tome I - Conifères; Tome II - Feuillus. - Nancy : Ecole nationale du Génie rural, des Eaux et des Forếts, 1984. - XV-88 p.; X-256 p. (3 édition en 1992).

KEIME (M.-P.). - Rôle des conditions climatiques et des propriétés du sol sur la croissance radiale du Chêne pédonculé, du Chêne sessile et du Hêtre. - Rennes: ENSA, 1985. - 36 p. + annexes (DAA).

LACHAUD (S.), MANSOURI (A.). - Reprise d'activité du cambium et xylogénèse chez l'Alisier torminal. Revue forestière française, vol. XLV, n 3, 1993, pp. 279-283.

LANIER (L.), RAMEAU (J.-C.), KELLER (R.), JOLY (H.-l.), DRAPIER (N.), SEVRIN (E.). - L'Alisier torminal Sorbus torminalis (L.) Crantz. - Revue forestière française, vol. XLII, $n^{\circ} 1,1990$, pp. 13-34.

LE GOFF (N.). - Indice de productivité des taillis-sous-futaie de Chêne dans la région Centre. - Annales des Sciences forestières, vol. $41, \mathrm{n}^{\circ} 1,1984$, pp. 1-34.

LE GOFF (N.). - Productivité du Frêne en rẻgion Nord-Picardie. A - Courbes de croissance en hauteur. Annales des Sciences forestières, vol. $39, \mathrm{n}^{\circ} 3,1982$, pp. 259-288.

LE GOFF (N.), MADESCLAIRE (A.). - Productivité des stations pour des essences disséminées en peuplement irrégulier. Le cas de l'Érable sycomore et du Merisier en taillis-sous-futaie sur les stations forestières des plateaux calcaires de Lorraine. In : Station forestière, production et qualité des bois : éléments méthodologiques. - CEMAGREF, 1989. - $254 \mathrm{p}$.

LEVVY (G.), BECKER (M.), DUHAMEL (D.). - A comparison of the ecology of pedunculate and sessile oaks : radial growth in the centre and northwest of France. - Forest Ecology and Management, 55, 1992, pp. 5163.

NICLOUX (C.). - Potentialités des stations torestières des plateaux calcaires de Lorraine et des marnes du Keuper du Plateau Lorrain pour l'Alisier torminal. Notes sur le Sorbier domestique. - Metz: Centre régional de la Propriété forestière Lorraine-Alsace, 1988. - V-61 p. (rapport de stage de $2^{\mathrm{e}}$ année ENGREF).

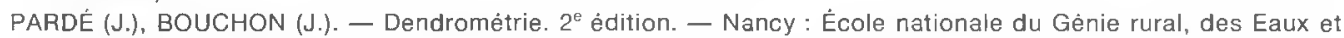
des Forêts, 1988 . - $328 \mathrm{p}$.

PARKER (R.E.). - Introductory statistics for biology. Second edition. - Institute of Biology; Edward Arnold Publishers Ltd, 1979 (Studies in Biology ; 43).

RAMEAU (J.-C.), MANSION (D.), DUMÉ (G.). - Flore forestière française. Guide ècologique illustré. Tome 1: Plaines et Collines. - Paris : Institut pour le Développement forestier, 1989. - $1785 \mathrm{p}$. 